\title{
Cómo realizar e interpretar una manometría esofágica de alta resolución
}

\section{How to Perform and Interpret High-resolution Esophageal Manometry}

Albis Hani, MD, ${ }^{1}$ Ana María Leguízamo, MD, ${ }^{1}$ Jhon Jaime Carvajal, MD, ${ }^{1}$ Gabriel Mosquera-Klinger, MD, ${ }^{1}$ Valeria Atenea Costa, MD. ${ }^{1}$

1 Hospital Universitario San Ignacio - HUSI, Servicio de Gastroenterología, Pontificia Universidad Javeriana. Bogotá, Colombia

Fecha recibido: $03-07-14$ Fecha aceptado: 02-02-15

\begin{abstract}
Resumen
Con la introducción de la manometría esofágica de alta resolución se revelaron patrones no identificados previamente de la función esofágica normal así como hallazgos anormales. De igual forma, adiciona patrones de presión gráficos y topografía de la presión esofágica, lo que lleva al desarrollo de nuevas herramientas para el análisis y clasificación de desórdenes motores esofágicos. En la actualidad la clasificación de Chicago es el método diagnóstico de análisis de los diferentes trastornos motores esofágicos; en Colombia, día a día vemos el crecimiento en la realización de este estudio. El artículo propone hacer una revisión de cómo realizar e interpretar una manometría esofágica de alta resolución.
\end{abstract}

\section{Palabras clave}

Desórdenes de motilidad esofágica, manometría esofágica de alta resolución, acalasia.

\begin{abstract} interpret high-resolution esophageal manometry.

Keywords

Esophageal motility disorders, esophageal manometry high resolution, achalasia.
\end{abstract}

The introduction of high-resolution esophageal manometry allowed physicians to identify both previously unidentified normal esophageal functions and patterns and various abnormalities. The creation of new charts of pressure patterns and the topography of esophageal pressure has led to the development of new tools for analysis and classification of esophageal motor disorders. At present, the Chicago classification is the diagnostic method of analysis used for various esophageal motor disorders. In Colombia, we see the use of high-resolution esophageal manometry growing on a daily basis. In this article we review how to perform and

\section{GENERALIDADES}

Durante varias décadas, la manometría esofágica ha sido el método de elección para evaluar trastornos motores esofágicos. La introducción de la manometría esofágica de alta resolución simplifica el estudio en la función motora del esófago (1).

La evaluación confiable de la función motora esofágica y gastrointestinal con técnicas manométricas llegó a ser posible en la década de 1970 cuando Wyle Jerry Dodds y Ron Arndorfer desarrollaron el primer sistema de manometría. Excepto por unas pocas modificaciones técnicas, su enfoque se mantuvo como estado del arte durante 2 décadas. En la década de 1990, Ray Clouse y sus colegas dieron nacimiento a la manometría de alta resolución al disminuir el espacio entre los sensores ubicados a lo largo del catéter de presión de la manometría convencional de $5 \mathrm{~cm}$ a $1 \mathrm{~cm}$, aumentando el número de sensores (1). 
Conceptualmente, la manometría de alta resolución se refiere al uso de un catéter con múltiples sensores de presión de alta fidelidad que sirven para capturar datos manométricos en un continuo espacial, sin las brechas sustanciales entre los sensores de presión típicos de la manometría convencional (2).

Algunos autores han utilizado el término "topografía de alta resolución de presión esofágica” para la manometría de alta resolución (3).

Con la manometría convencional no es posible ver simultáneamente la función motora del esfínter esofágico superior (EES), del cuerpo del esófago y del esfínter esofágico inferior (EEI) con cada deglución, mientras que la manometría de alta resolución brinda esta posibilidad con una completa representación espacio-temporal de la función motora del esófago (1).

La utilidad de la manometría de alta resolución es superar las limitaciones de los sistemas de la manometría esofágica convencional a través de tecnologías electrónicas avanzadas. La clave de este desarrollo está determinada por el aumento en el número de sensores de presión en el catéter del equipo de alta resolución, 36 en total, los cuales se encuentran a una distancia entre ellos menor de $2 \mathrm{~cm}$. Esto permite evaluar la presión intraluminal a través de toda la extensión del esófago y en los esfínteres. Cada sensor tiene sensibilidad circunferencial (3).

Igualmente, la manometría de alta resolución ha mejorado la habilidad de predecir el fallo o el éxito en el movimiento del bolo a través el esófago comparado con la manometría convencional, y hasta la ocurrencia de eventos de reflujo. Además, los componentes de la barrera antirreflujo y su interacción dinámica pueden ser muy bien diferenciados. Los registros de la manometría de alta resolución revelan la compleja anatomía funcional del esófago y sus esfínteres, y permiten evaluar con mayor exactitud que los equipos de manometría convencional el monitoreo de la presión del esfínter esofágico inferior y el reconocimiento de relajaciones espontáneas transitorias del mismo (4).
La presentación de los datos de presión con contornos de color o presiones esofágicas a través de topografía condujo al desarrollo de nuevas herramientas para analizar y clasificar los patrones motores del esófago. El enfoque actual estándar para hacer esto es la clasificación de Chicago (1).

\section{Diferencias con la manometría convencional}

La manometría esofágica de alta resolución revela la acción dinámica del esfínter esofágico superior, el carácter segmentario de la peristalsis esofágica y la anatomía funcional de la unión gastroesofágica. En segundo lugar, los gráficos espacio-temporales construidos a partir de los datos obtenidos por el sensor de presión proporcionan una representación exacta de la relación entre la fuerza de cierre (fuerza contráctil), la fuerza de aclaramiento y la resistencia al flujo (nadir de presión y gradiente de presión a través de la unión esofagogástrica). Todos estos factores son necesarios para la apreciación completa de la biomecánica del transporte del bolo (4).

Una ventaja es que disminuye el tiempo necesario para el estudio ya que la colocación del catéter es rápida y fácil (tiempo promedio del procedimiento 8,2 versus 24,4 minutos en la convencional, $\mathrm{p}<0,0001)(3,4)$. Otra clara ventaja de la manometría de alta resolución es la eliminación de la necesidad de reposicionar el catéter durante el curso del estudio mediante la técnica "pull through" de la manometría convencional, para así determinar las características del esfínter esofágico inferior y de la unión gastroesofágica. Esto ha resultado en una mejoría significativa en la objetividad de la evaluación de la unión gastroesofágica tanto en reposo como en respuesta a la deglución (tabla 1) $(2,3)$.

Las desventajas de la manometría de alta resolución son el alto costo del equipo y la falta de experiencia en la interpretación de los registros espacio-temporales porque trae el riesgo de sobrediagnóstico de registros de dismotilidad esofágica insignificante (tabla 1) (4).

Tabla 1. Comparación de los métodos manométricos esofágicos.

\begin{tabular}{llll}
\hline & $\begin{array}{c}\text { Manometría convencional con } \\
\text { maniobra de "pull through" }\end{array}$ & $\begin{array}{c}\text { Manometría convencional tipo } \\
\text { manga }\end{array}$ & $\begin{array}{c}\text { Manometría de alta } \\
\text { resolución }\end{array}$ \\
\hline Costo & Bajo & Bajo & Alto \\
Ejecución & Procedimiento elaborado y con & Procedimiento elaborado y con & Relativamente simple y rápido \\
& consumo moderado de tiempo & consumo moderado de tiempo & Relativamente fácil \\
Interpretación & Requiere experiencia & Requiere experiencia & Sí \\
Medición de presión EEl y relajación & Limitada & Sí & Sí \\
Medición de EES y relajación & No & Limitada & \\
\hline
\end{tabular}

EEI: esfínter esofágico inferior; EES: esfínter esofágico superior. 


\section{INDICACIONES DE LA MANOMETRÍA ESOFÁGICA DE ALTA RESOLUCIÓN}

Las indicaciones para realizar una manometría esofágica de alta resolución son las mismas que existen para realizar un estudio convencional, con algunas ventajas y detalles en la valoración de aspectos como los esfínteres esofágicos superior e inferior y el transporte del bolo (5). Es así como la principal indicación para realizar una manometría esofágica de alta resolución es el estudio de una disfagia cuya causa no haya podido ser establecida por estudios endoscópicos o imagenológicos, y se sospeche una alteración motora esofágica $(5,6)$. Otra indicación para realizar manometría esofágica es el estudio de dolor torácico de origen no cardiaco. Diagnósticos como esófago en cascanueces, Jackhammer y el espasmo esofágico difuso pueden explicar estos cuadros clínicos $(5,7)$.

La manometría esofágica es el patrón de oro para el diagnóstico de acalasia, por lo que ante la sospecha de la misma es imperativo realizar este estudio. Con el advenimiento de la manometría esofágica de alta resolución se ha podido subclasificar esta patología y de esta manera se ha demostrado cuáles subtipos pueden tener mejor respuesta al tratamiento $(6,7)$. También se ha descrito su utilidad en el estudio de trastornos de la motilidad esofágica en enfermedades que comprometen el tejido conectivo como la esclerosis sistémica, donde el compromiso esofágico alcanza hasta el 90\% de los pacientes con esta condición. En ésta los hallazgos de la manometría esofágica son hipotonía del esfínter esofágico inferior, peristalsis infectiva y aperistalsis (5-7).

En el contexto preoperatorio de un paciente que va a ser llevado a cirugía antirreflujo, es importante para descartar previamente la presencia de una alteración motora como la acalasia. También dentro de los grupos quirúrgicos se ha descrito la utilidad de la manometría en el momento de tomar la decisión del tipo de cirugía a realizar, como una funduplicatura de 180 o 360 grados. De igual forma, en el cuadro de un paciente con disfagia posoperatoria está descrito el uso de manometría esofágica de alta resolución con el fin de establecer un plan de tratamiento posterior $(5,7)$.

En el posicionamiento de catéter de pHmetría esofágica se ha descrito la previa ubicación del esfínter esofágico inferior mediante manometría esofágica, la cual es utilizada por varios grupos de gastroenterólogos que realizan estudios de fisiología esofágica (5).

\section{CÓMO SE REALIZA LA MANOMETRÍA ESOFÁGICA DE ALTA RESOLUCIÓN}

En el momento de realizar una manometría esofágica de alta resolución, es importante tener en mente varios puntos, los cuales se muestran a continuación.

\section{Preparación del paciente}

Idealmente se debe indicar al paciente un ayuno de 6 horas o 12 horas si se sospecha un diagnóstico de acalasia, esto teniendo en cuenta el riesgo de broncoaspiración por la posibilidad de tener contenido alimentario en la luz esofágica por la alteración motora $(5,6)$.

\section{Uso de medicamentos que puedan alterar la motilidad esofágica}

El día del examen debe indicarse la suspensión de medicamentos como bloqueadores de los canales del calcio, nitratos, procinéticos, loperamida, antagonistas de receptores $\beta$, opiáceos y anticolinérgicos. Estos solo deben ser utilizados si su suspensión conlleva a una alteración en el bienestar del paciente (5).

\section{Explicación acerca del procedimiento y firma de consentimiento informado}

Ya que se trata de un estudio invasivo, debe dársele al paciente una detallada explicación acerca de la técnica aplicada en el procedimiento y las posibles incomodidades que va a experimentar. Una vez el paciente haya aprobado realizar el procedimiento, se sugiere realizar la firma del consentimiento informado (6).

\section{Preparación del equipo}

Previo a realizar la intubación esofágica del paciente debe hacerse una calibración, la cual varía de acuerdo con el tipo de sonda que se va a utilizar. Debe asegurarse una limpieza del catéter conforme con los estándares aceptados a nivel mundial para evitar la transmisión de infecciones a través del dispositivo $(5,7)$.

\section{Realizar el estudio}

El posicionamiento de la sonda debe realizarse por vía transnasal. Una vez se logre la canulación se realizará el posicionamiento del catéter, el cual quedara fijado de acuerdo con el tipo de equipo utilizado según la estatura (Sierra ${ }^{\circ}$ o, si se utilizan otros equipos, hasta identificar el esfínter esofágico inferior y superior en la gráfica (figura 1) $(1,5,6)$.

Una vez se encuentra en posición se debe iniciar con la medición de las presiones basales de los esfínteres. Para esto se pide al paciente que no realice degluciones por espacio de 30 segundos. Tan pronto se haga la medición, se puede continuar el estudio dándole al paciente tragos de $5 \mathrm{~mL}$ de agua, para un total de 10 degluciones. Recientemente se ha propuesto que una vez se completen las 10 degluciones, 
como parte del estudio, se añada la evaluación de degluciones múltiples, esto dado el concepto reciente de integridad neuromuscular. Sin embargo, este último no está estandarizado, por lo que su aplicación depende de la preferencia del grupo evaluador que realice el estudio (8).

\section{CÓMO INTERPRETAR LA MANOMETRÍA DE ALTA RESOLUCIÓN}

La manometría de alta resolución demuestra la presión de reposo de los esfínteres y la actividad motora esofágica desencadenada por las degluciones. La mayoría de los análisis son generados por el software del equipo, sin embargo, los algoritmos no son perfectos por lo que es imprescindible que se revisen cada una de las degluciones para asegurar que los parámetros y mediciones sean apropiados.

El estudio se inicia con la evaluación de las presiones de reposo EES y del EEI, que son identificadas como 2 zonas con aumento de presión fácilmente evidenciada por un cambio de color en la topografía esofágica (figura 2).

La manometría de alta resolución permite diferenciar la presión basal del esfínter esofágico inferior de la contracción diafragmática crural. En condiciones normales estas 2 zonas de presión deben coincidir, su separación indica la presencia de una hernia diafragmática (7). Asimismo se puede identificar el punto de inversión de presión (PIP), que es el lugar donde se da el cambio de una presión negativa generada por la presión intratorácica a una presión positiva generada por la presión intragástrica; este punto señala la división que genera el diafragma entre el tórax y el abdomen (figura 3).

Cuando nos enfrentamos al análisis de una manometría esofágica de alta resolución es conveniente realizar una interpretación por etapas, donde inicialmente se evalúa el EEI y su relajación y luego se evalúan los parámetros de presión topográfica esofágica durante cada deglución para definir los diferentes trastornos de motilidad esofágica (duración, velocidad y amplitud) (9).

El primer paso es identificar la presión basal del EEI y si existe o no una hernia hiatal. Esto se logra evidenciando el punto de inversión de presión (PIP) el cual, en condiciones normales, se debe encontrar inmediatamente por encima del borde proximal del esfínter esofágico inferior; esto significa que la presión generada por el EEI y la presión del diafragma crural coinciden (figura 2).

Luego debe definirse si la relajación del EEI es normal o no durante la deglución. En la manometría de alta resolución la relajación del EEI se logra evaluar con la presión integrada de relajación (IRP, por sus siglas en inglés). Para medir la IRP el equipo toma 10 segundos a partir del inicio de la deglución (esto es cuando se inicia la relajación del EES). La IRP es la presión promedio más baja en la unión esofagogástrica durante 4 de los 10 segundos mencionados, que pueden ser continuos o discontinuos en una deglución (figura 4). Una IRP mayor de $15 \mathrm{~mm} \mathrm{Hg}$ significa aumento en la resistencia al tránsito del bolo en la unión esofagogástrica y se considera patológica (10). Cualquier proceso patológico, ya sea mecánico o funcional, que impida el paso en la unión esofagogástrica puede aumentar el IRP (1). Algunas de estas alteraciones pueden ser la acalasia y la obstrucción del tracto de salida (neoplasias, estenosis benignas y complicaciones de la funduplicatura) (figura 5).

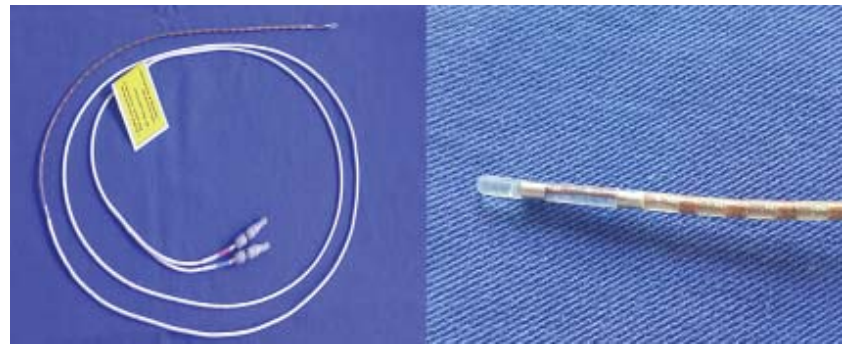

Figura 1. A. Catéter de manometría de alta resolución. B. Sensores del catéter.

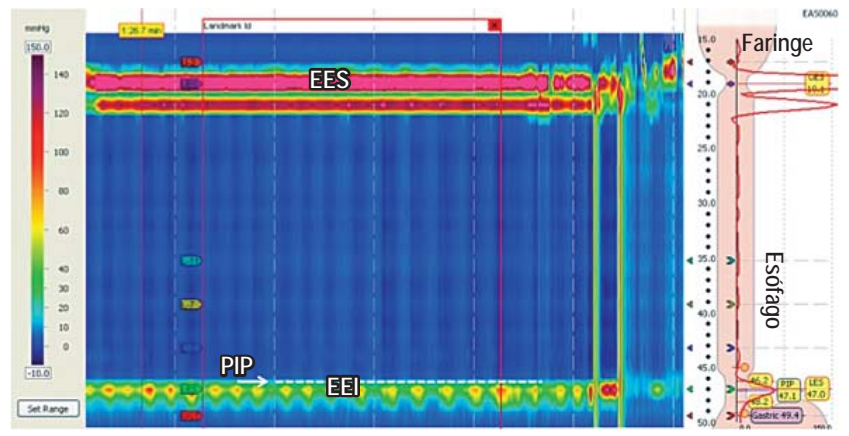

Figura 2. Evidencias de zonas de presión generadas por el EES y el EEI. EEI: esfínter esofágico inferior; EES: esfínter esofágico superior; PIP: punto de inversión de presión.

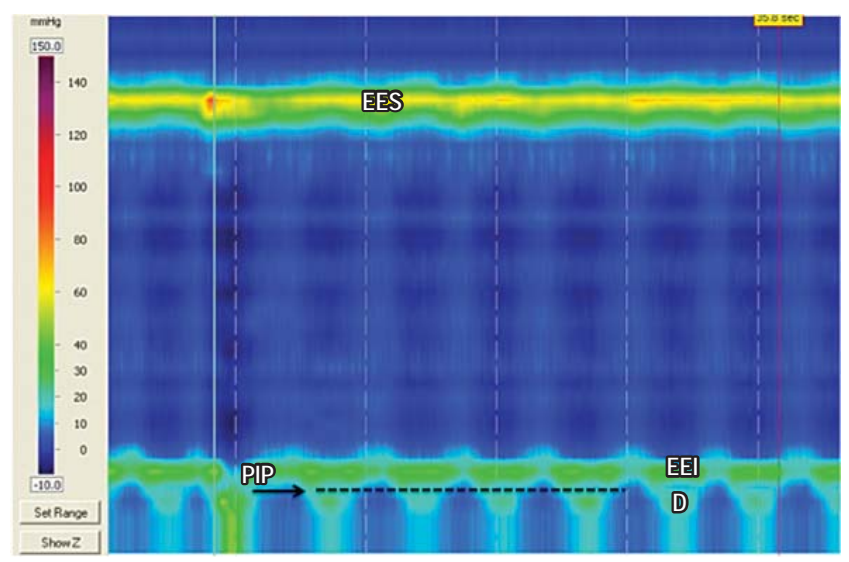

Figura 3. Evidencia de 2 zonas de presión que indican el EEI y la impresión del diafragma crural (D), así como el PIP. EEI: esfínter esofágico inferior; EES: esfínter esofágico superior; PIP: punto de inversión de presión. 


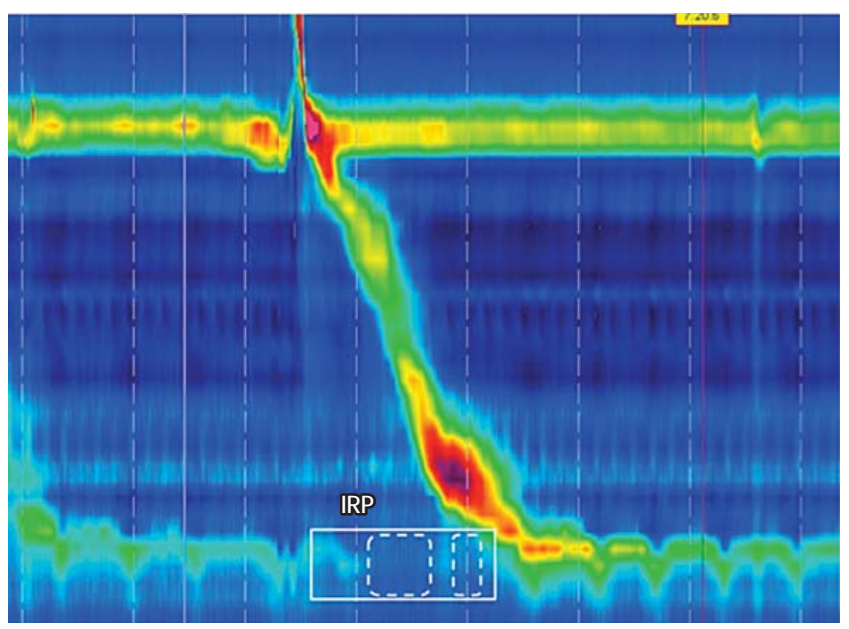

Figura 4. Análisis de la relajación del EEI. El IRP es la medición de la presión más baja en el EEI durante la deglución, tiene en cuenta 4 segundos que pueden ser continuos o discontinuos (líneas discontinuas).

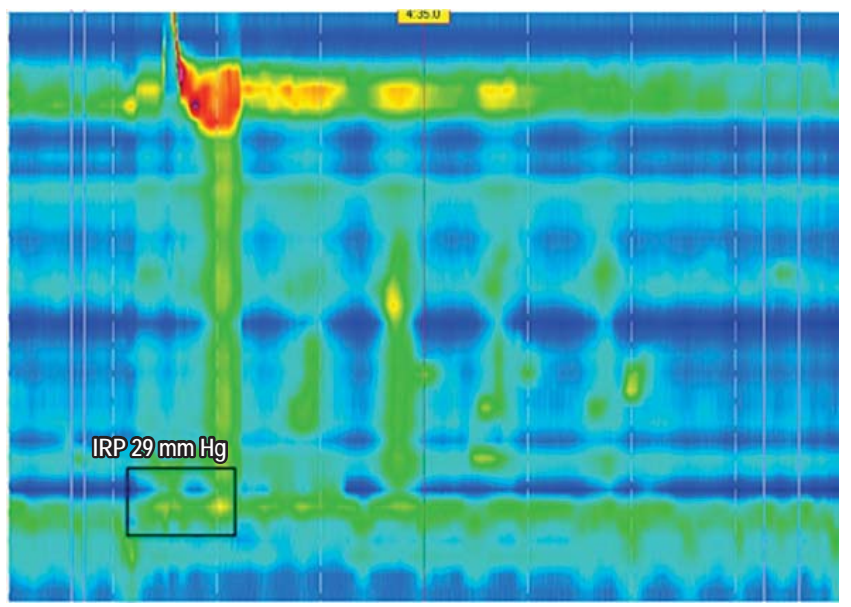

Figura 5. Ausencia de relajación del esfínter esofágico inferior en un paciente con acalasia. Nótese además la ausencia de onda peristáltica.

Luego se determina si la actividad peristáltica es normal, fallida o si existen zonas de presión en el contorno isobárico menores de $20 \mathrm{~mm} \mathrm{Hg}$ y que se prolonguen por $2-5 \mathrm{~cm}$ (cortas) o por más de $5 \mathrm{~cm}$ (largas). Estas zonas se conocen como defectos peristálticos. Se eligió el valor límite de $20 \mathrm{~mm} \mathrm{Hg}$ como la presión mínima necesaria para el tránsito del bolo cuando la unión esofagogástrica funciona adecuadamente (1).

Estos defectos peristálticos son más significativos si se presentan en la porción más distal del esófago, pues con frecuencia se encuentra una disminución de la presión en la unión del tercio proximal con el medio donde se encuentra la zona de transición de músculo estriado a músculo liso; sin embargo, esta disminución en la presión no debe ser de gran longitud (figuras 6-8).

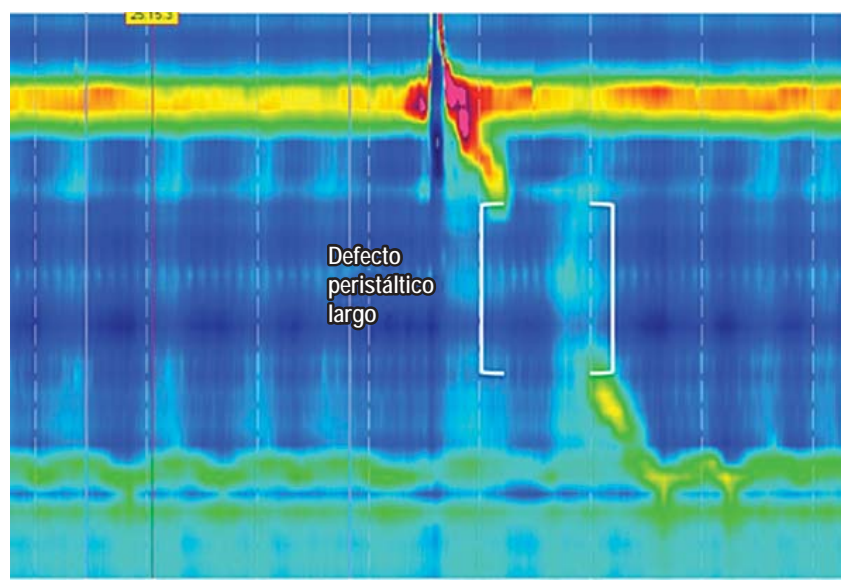

Figura 6. Evaluación de la integridad de la peristáltica. Se muestra un defecto peristáltico mayor de $5 \mathrm{~cm}$.

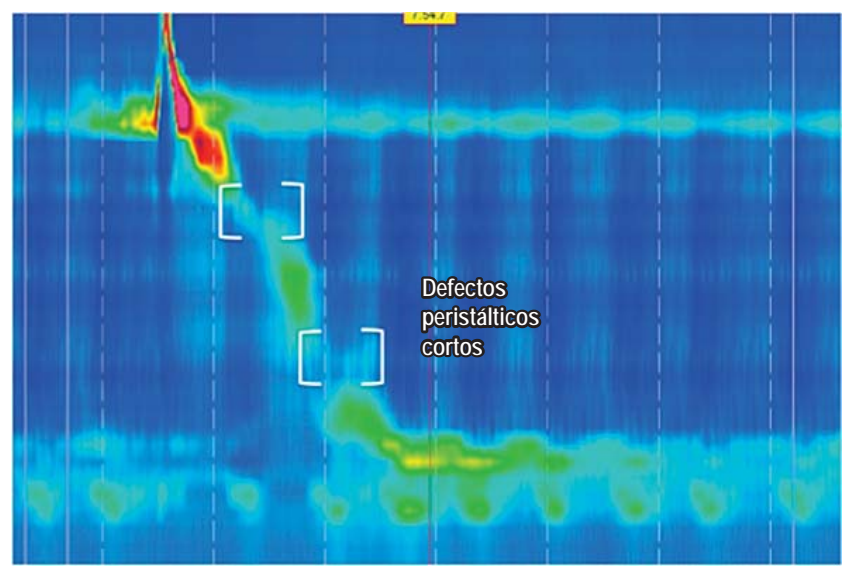

Figura 7. Evaluación de la integridad de la actividad peristáltica. Se muestran defectos peristálticos menores de $5 \mathrm{~cm}$.

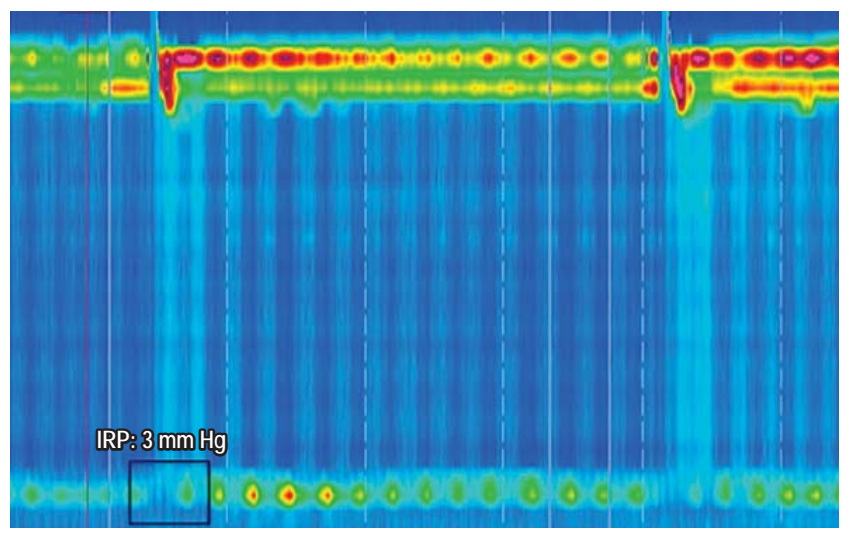

Figura 8. Evaluación de la integridad de la actividad peristáltica. Ausencia de onda peristáltica denominada "onda fallida".

El punto de desaceleración contráctil (CDP, por sus siglas en inglés) es el lugar donde existe una desaceleración en la velocidad del frente de la onda peristáltica, se localiza en el tercio 
distal del esófago cerca al esfínter esofágico inferior (11). Generalmente está asociado al punto de mayor contracción axial del esófago. Funcionalmente, el CDP es el punto en el tiempo donde la onda peristáltica termina y el EEI inicia el descenso a su posición de reposo (10) (figura 9).

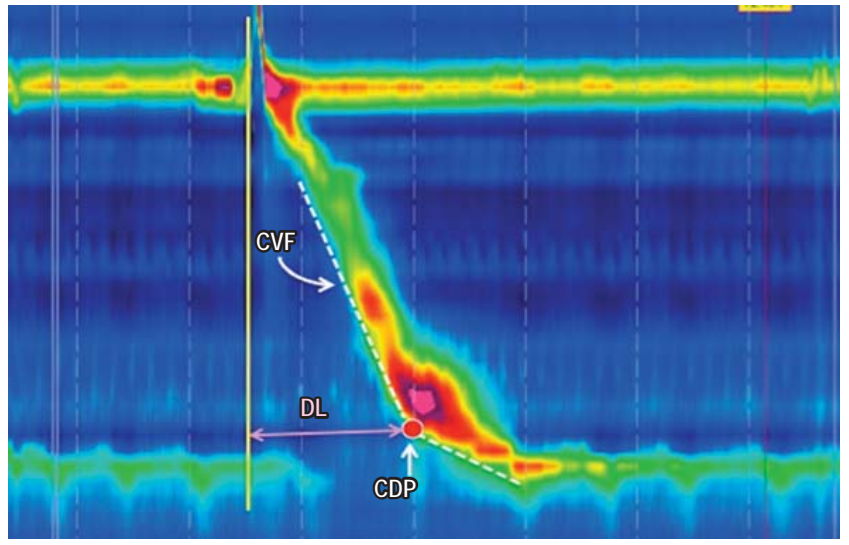

Figura 9. Punto de desaceleración contráctil (CDP). Es el sitio donde hay disminución de la velocidad del frente de onda. Velocidad del frente contráctil (CVF). El tiempo de latencia distal (DL) se mide entre el inicio de la relajación del EES y el punto de desaceleración contráctil (CDP).

Propagación de la onda peristáltica: el tiempo de propagación de la onda peristáltica se determina por la latencia distal (DL, por sus siglas en inglés) que indica si la contracción es prematura y si existe alteración en la inhibición normal del cuerpo esofágico que regula la velocidad de propagación de la onda (12). La latencia distal se obtiene a partir del intervalo de tiempo que transcurre entre el inicio de la relajación del EES y el punto de desaceleración contráctil (CDP); su límite inferior es 4,5 segundos (13) (figura 9). La velocidad de la onda peristáltica es medida mediante la velocidad del frente contráctil ( $\mathrm{CFV}$, por sus siglas en inglés). El equipo calcula la pendiente a partir de una línea que va entre la zona de transición y el punto de desaceleración contráctil (CDP) y su valor normal no debe exceder los $9 \mathrm{~cm} / \mathrm{s}$ (11) (figura 9).

Fuerza contráctil: la riqueza del detalle generado por la manometría de alta resolución favorece una mejor medida de la fuerza contráctil del esófago permitiendo la generación de un valor definido que tiene en cuenta la presión, el tiempo y la distancia en los dos tercios distales del esófago (14). Esta medida se denomina la integral de contractilidad distal (DCI, por sus siglas en inglés), valor que se genera con base en la amplitud de contracción promedio generada por el músculo liso, la duración de la contracción y la distancia de propagación de la onda entre la zona de transición y la porción más proximal de la unión esofagogástrica. $\mathrm{Su}$ valor normal es $<5000 \mathrm{~mm} \mathrm{Hg} x \mathrm{~cm} x \mathrm{~s}$ (15) (figura 10).

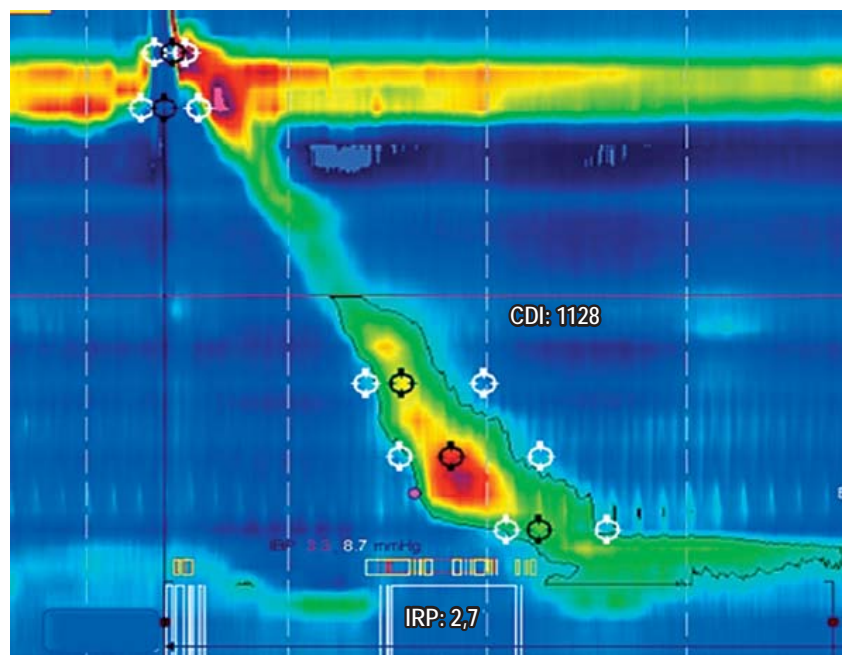

Figura 10. Integral de contractilidad distal (CDI). Corresponde a la amplitud de contracción promedio dentro del contorno isobárico (línea negra continua).

Finalmente se determinan los patrones de presurización anormal. La presión intrabolo anormalmente alta (denominada patrón de presurización) es un signo de alteración en la mecánica del tránsito del bolo y puede ser secundaria a obstrucción del flujo o alteración en la distensibilidad de la pared esofágica. La manometría de alta resolución permite evidenciar patologías que causen obstrucción del tránsito del bolo en la unión esofagogástrica como tumores, estenosis benignas, funduplicaturas apretadas y algunas variantes de acalasia; o alteraciones obstructivas en el esfínter esofágico superior como la barra y acalasia del cricofaríngeo. Se identifica por una zona de presión constante (presión isobárica) de longitud variable en la porción distal del esófago o en el segmento proximal al cricofaríngeo $(7,10)$ (figura 11).
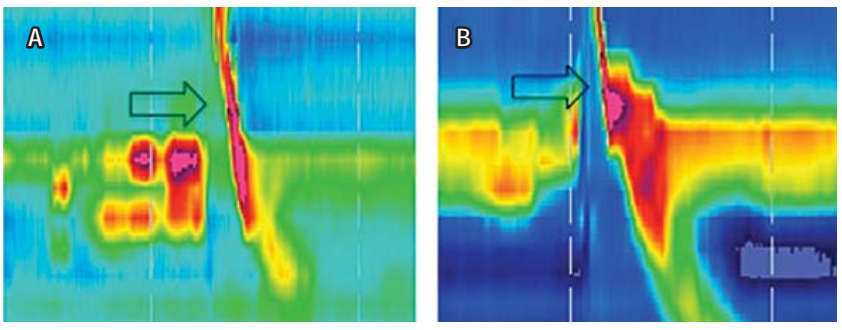

Figura 11. A. Patrón de presurización anormal en el cricofaríngeo que demuestra una presión intrabolo aumentada en un paciente con barra cricofaríngea. B. Presión intrabolo normal.

Luego de analizar cada una de las degluciones con las herramientas antes mencionadas se procede a utilizar la clasificación de Chicago para el diagnóstico más apropiado de los trastornos motores esofágicos (15) (tabla 2). 
Tabla 2. Valores normales en la manometría de alta resolución esofágica.

\begin{tabular}{ll}
\hline \multicolumn{1}{c}{ Característica } & \multicolumn{1}{c}{ Valor normal } \\
\hline Relajación del EEI & $\mathrm{IRP}<15 \mathrm{~mm} \mathrm{Hg}$ \\
Propagación peristáltica & $\mathrm{CVF}<9 \mathrm{~cm} / \mathrm{s}$ \\
& $\mathrm{DL}>4,5 \mathrm{~s}$ \\
Vigor contráctil & $\mathrm{DCl} 450-5000 \mathrm{~mm} \mathrm{Hg} \times \mathrm{s} \times \mathrm{cm}$ \\
\hline
\end{tabular}

Presión integrada de relajación (IRP, por sus siglas en inglés), latencia distal (DL, por sus siglas en inglés), velocidad del frente contráctil (CFV, por sus siglas en inglés), integral de contractilidad distal (DCI, por sus siglas en inglés).

\section{Alteraciones manométricas según la clasificación de Chicago}

La clasificación de Chicago se utiliza para catalogar los trastornos motores esofágicos; para la interpretación de ésta debemos revisar, en primer lugar, la competencia del esfínter esofágico inferior, y además caracterizar la función motora esofágica. Vale aclarar que en la clasificación de
Chicago se tienen en cuenta exclusivamente la motilidad del esófago distal y el esfínter esofágico inferior.

De acuerdo con esto, se revisarán 4 grandes grupos de anormalidades motoras esofágicas: acalasia, obstrucción esofágica, función motora esofágica anormal y función motora esofágica limítrofe o borderline (que pueden verse en pacientes asintomáticos) (figura 12).

Uno de los principales patrones y trastornos motores esofágicos es la acalasia, que es clínica y fisiopatológicamente el trastorno más entendido. La acalasia ha sido definida por muchos años mediante manometría convencional como falla en la relajación del esfínter esofágico inferior y ausencia de peristaltismo en el músculo liso esofágico. Con la introducción de la manometría esofágica de alta resolución, el diagnóstico se ha subdividido en 3 subtipos (7) que se caracterizan por falla de la relajación del esfínter esofágico inferior pero con diferentes patrones motores esofágicos. En la acalasia tipo I no es apreciable la actividad motora esofágica, es la que previamente se describía como clásica con contracciones simultáneas de baja amplitud, la

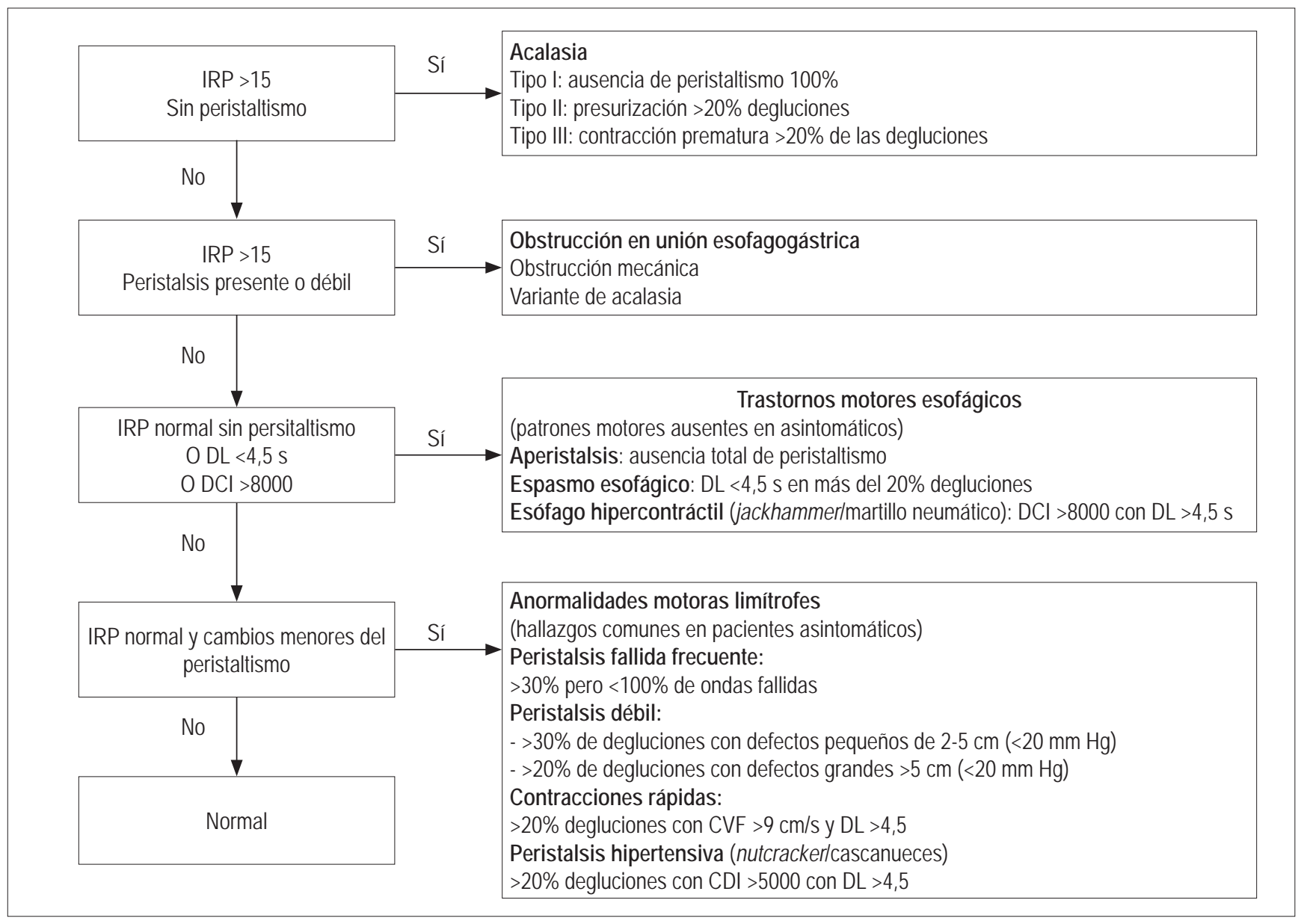

Figura 12. Flujograma diagnóstico de los trastornos motores esofágicos. Modificada de la referencia 1. CFV: velocidad del frente contráctil; DCI: integral de contractilidad distal; DL: latencia distal; IRP: presión integrada de relajación. 
acalasia tipo II se caracteriza por presurización panesofágica en más del $20 \%$ de los tragos, y en la acalasia tipo III se observan contracciones prematuras (espásticas) en más del $20 \%$ de las degluciones.

$\mathrm{Al}$ revisar la evidencia respecto a los resultados del tratamiento con base en el tipo de acalasia se ha demostrado que la tipo I (figura 13) responde mejor al tratamiento con miotomía de Heller que con dilatación con balón, el tipo II es muy sensible a cualquier tratamiento elegido (figura 14), y el tipo III tiene el peor pronóstico terapéutico $(16,17)$.

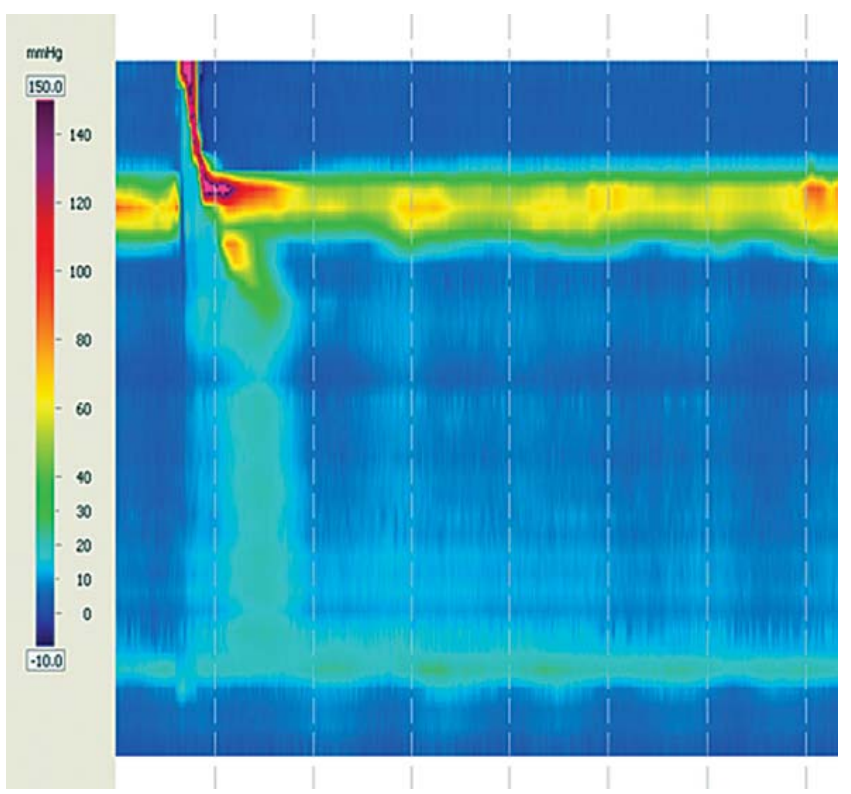

Figura 13. Acalasia tipo I.

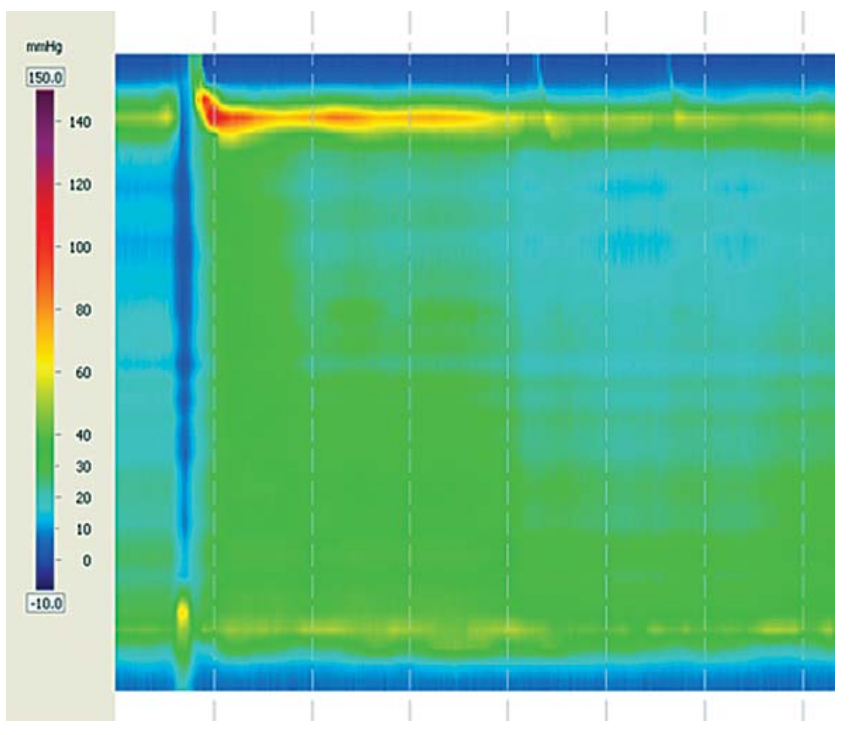

Figura 14. Acalasia tipo II.
La clasificación de función motora esofágica anormal es un grupo de diversas anormalidades motoras esofágicas que no ocurren en individuos asintomáticos. Entre estos trastornos se encuentran el esófago en cascanueces (Nutcracker), esófago en martillo neumático (esófago de Jackhammer), aperistalsis y espasmo esofágico distal (1).

La peristalsis hipertensiva se define como ondas peristálticas en el músculo liso esofágico con DCI entre 5000 y $8000 \mathrm{~mm} \mathrm{Hg} / \mathrm{cm} / \mathrm{s}$ e IRP normal. Estos pacientes pueden tener disfagia y/o dolor torácico no cardiogénico. Incluye el esófago en cascanueces y el esófago de Jackhammer.

El esófago en cascanueces se define como 2 o más degluciones que producen una contracción propagada en el músculo liso con un DCI mayor de $5000 \mathrm{~mm} \mathrm{Hg} / \mathrm{cm} / \mathrm{s}$, con una DL y una IRP normales (figura 15).

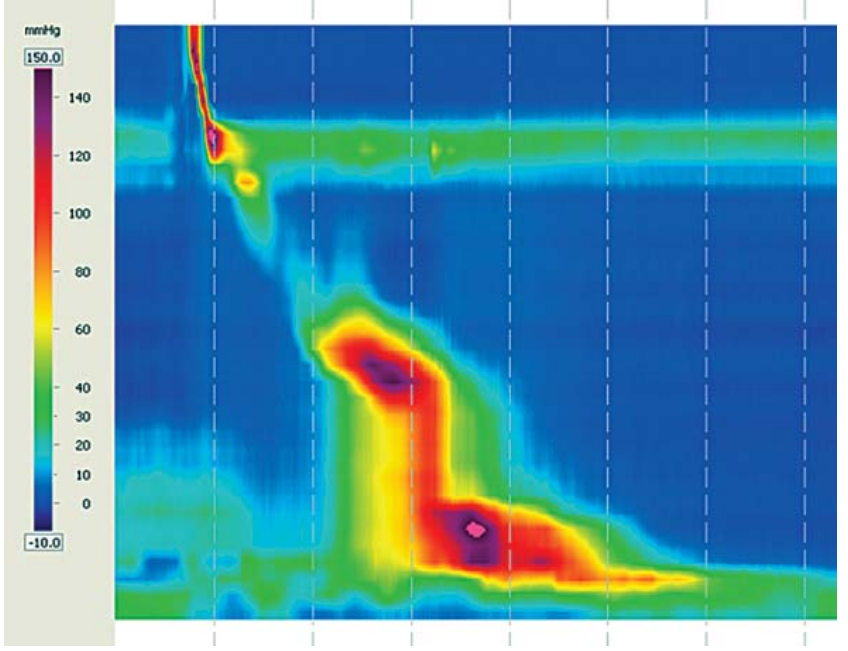

Figura 15. Esófago de cascanueces.

El esófago en "Jackhammer" es un patrón relativamente raro que se define como 1 o más degluciones que producen una contracción propagada en el musculo liso con un DCI mayor de $8000 \mathrm{~mm} \mathrm{Hg} / \mathrm{cm} / \mathrm{s}$, con una DL y una IRP normales (figura 16).

La aperistalsis se caracteriza por una presión de relajación integrada (IRP) normal y ninguna onda peristáltica en el esófago distal. Siempre que se vea este patrón manométrico se debe preguntar si el paciente tiene alguna alteración, ya sea esclerodermia, diabetes o hipotiroidismo (figura 17).

El espasmo esofágico distal es un patrón topográfico infrecuente que se caracteriza por menos del $20 \%$ de degluciones con latencia distal (DL) corta (menor de 4,5 segundos) y con IRP normal. Estas contracciones suelen ser prolongadas y multifásicas. El patrón típicamente está asociado con disfagia y/o dolor torácico no cardiogénico, los cuales podrían ser susceptibles a manejo con analgésicos viscera- 
les y/o relajantes de músculo liso/toxina botulínica. Esta combinación de patrones podría ser también el signo inicial de una neuropatía mientérica inhibitoria y podría convertirse en acalasia en el futuro.

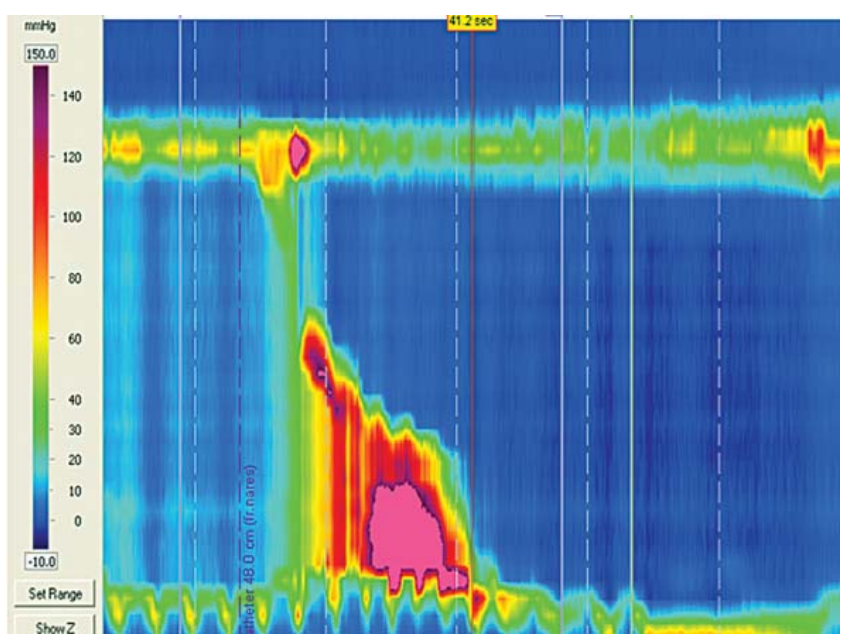

Figura 16. Trastorno hipercontráctil tipo jackhammer.

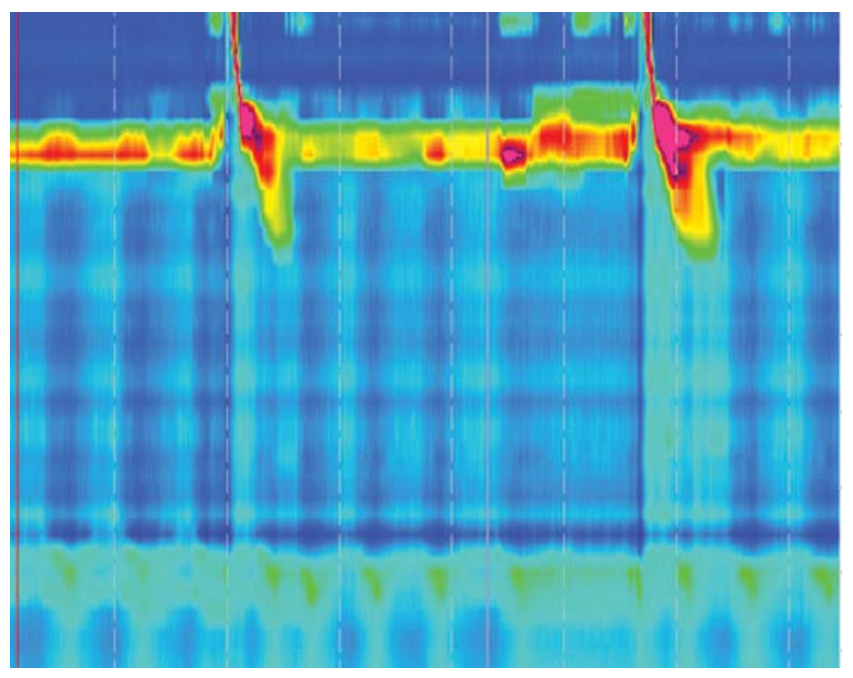

Figura 17. Aperistalsis.

La función motora esofágica limítrofe o borderline es un grupo heterogéneo de alteraciones motoras que incluyen el peristaltismo débil y contracciones rápidas. Probablemente representan la mayor parte de anormalidades manométricas observadas en múltiples laboratorios de motilidad.

El peristaltismo débil se caracteriza por defectos en el contorno isobárico de $20 \mathrm{~mm} \mathrm{Hg}$ y peristalsis fallidas frecuentes, son similares a la peristalsis inefectiva descrita en la manometría convencional. Estas anormalidades motoras están asociadas con pobre tránsito del bolo y escape proximal, principalmente cuando la apertura está a nivel de la zona de transición $(1,18)$. La contracción rápida se caracteriza por más del $20 \%$ de tragos con contracciones peristálticas con una velocidad mayor de $9 \mathrm{~cm} / \mathrm{s}$, con DL y IRP normales.

\section{CONCLUSIONES}

La manometría esofágica de alta resolución proporcionó por primera vez una visión completa espacial y temporal de la función motora del esófago (1). De hecho, casi todos los trastornos motores del esófago pueden producir diferentes patrones de topografía de presión esofágica que se reconocen fácilmente (1).

Serios esfuerzos para identificar distintos trastornos motores del esófago condujeron al desarrollo de la Clasificación de Chicago, que sigue siendo la más utilizada actualmente para la interpretación de los mismos (1).

Uno de los objetivos de un esquema diagnóstico a través de la clasificación de Chicago es la catalogación exacta de los pacientes para su inclusión en diversos ensayos clínicos, y en última instancia para ayudar a determinar los tratamientos específicos de la enfermedad (20).

Todas las imágenes fueron extraídas del equipo de manometría de alta resolución de la Unidad de Gastroenterología del Hospital Universitario San Ignacio. Bogotá, Colombia.

\section{REFERENCIAS}

1. Conklin JL. Evaluation of Esophageal Motor function with high-resolution manometry. J Neurogastroenterol Motil 2013;19(3):281-94.

2. Bansala A, Kahrilas P. Has high-resolution manometry changed the approach to esophageal motility disorders? Curr Opin Gastroenterol 2010;26(4):344-51.

3. Roman P, Kahrilas P. Challenges in the swallowing mechanism; non-obstructive dysphagia in the era of high-resolution manometry and impedance. Gastroenterology Clin N Am 2011;40:823-35.

4. Fox MR, Bredenoord AJ. Oesophageal high-resolution manometry: Moving from research into clinical practice. Gut 2008;57:405-23.

5. Murray J, Clouse R, Conklin J. Components of the standard esophageal manometry. Neurogastroenterol Motil. 2003; 15:591-606.

6. Kharilas P, Sifrim D. High-resolution manometry and impedance-pH/manometry: valuable tools in clinical and investigational esophagology. Gastroenterology 2008;135:756-69.

7. Conklin JL, Pimentel M, Soffer E. A color atlas of high-resolution manometry. Springer; 2009.

8. Fornari F, Bravi I. Multiple rapid swallowing: A complementary test during standard esophageal manometry. Neurogastroent Motil 2009;21:718.

9. Dustin A, Pandolfino J. High-resolution manometry and esophageal pressure topography. Filling the gaps of convention manometry. Gastroenterl Clin N Am 2013;62:1-15. 
10. Ghosh SK, Pandolfino JE, Rice J, Clarke JO, Kwiatek M, Kahrilas PJ. Impaired deglutitive EGJ relaxation in clinical esophageal manometry: A quantitative analysis of 400 patients and 75 controls. Am J Physiol Gastrointest Liver Physiol 2007;293:878-85.

11. Pandolfino JE, Leslie E, Luger D, Mitchell B, Kwiatek MA, Kahrilas PJ. The contractile deceleration point: An important physiological landmark on oesophageal pressure topography. Neurogastrenterol Motil 2010;22:395-400.

12. Behar J, Biancani P. Pathogenesis of simultaneous esophageal contractions in patients with motility disorders. Gastroenterology 1993;105:111-8.

13. Roman S, Lin Z, Pandolfino JE, Kahrilas PJ. Distal contraction latency: A measure of propagation velocity optimized for esophageal pressure topography studies. Am J Gastroenterol 2011;106:443-51.

14. Pandolfino JE, et al. Distal esophageal spasm in high-resolution esophageal pressure topography: Defining clinical phenotypes. Gastroenterology 2011;141:469-75.

15. Gyawali CP, et al. Evaluation of esophageal motor function in clinical practice. Neurogastroenterol Motil 2013;25:99133.
16. Pandolfino JE, Kwiatek MA, Nealis T, Bulsiewicz W, Post J, Kahrilas PJ. Achalasia: A new clinically relevant classification by high-resolution manometry. Gastroenterology 2008; 135:1526-33.

17. Salvador $\mathrm{R}$, et al. The preoperative manometric pattern predicts the outcome of surgical treatment for esophageal achalasia. J Gastrointest Surg 2010;14:1635-45.

18. Pandolfino JE, Zhang QG, Ghosh SK, Han A, Boniquit C, Kahrilas PJ. Transient lower esophageal sphincter relaxations and reflux: Mechanistic analysis using concurrent fluoroscopy and high-resolution manometry. Gastroenterology 2006; 131:1725-33.

19. Ravi K, Friesen L, Issaka RB, Kahrilas PJ, Pandolfino JE. The natural history of patients with normal and borderline motor function on high-resolution manometry. Gastroenterology 2012;12(Suppl 1):S34.

20. Carlson D, Pandolfino J. The Chicago Criteria for esophageal motility disorders: What has changed in the past 5 years? Curr Opin Gastroenterol 2012;28(4):395-402. 